\title{
Sedative and Immunosuppressive Effects of Dexmedetomidine in Transplantation
}

\author{
Chen-Fang Lee ${ }^{1,2, *} \mathbb{D}$, Chih-Hsien Cheng ${ }^{1}$, Hao-Chien Hung ${ }^{1}{ }^{(\mathbb{D}}$, Jin-Chiao Lee ${ }^{1}$, Yu-Chiao Wang ${ }^{1}{ }^{(D}$, \\ Tsung-Han $\left.\mathrm{Wu}^{1}{ }^{1}{ }^{(}\right)$, Ting-Jung $\mathrm{Wu}^{1}{ }^{1}$, Hong-Shiue Chou ${ }^{1}$, Kun-Ming Chan ${ }^{1}{ }^{\mathbb{D}}$ and Wei-Chen Lee ${ }^{1}$ \\ 1 Department of Liver and Transplantation Surgery, Chang-Gung Memorial Hospital at Linkou, \\ Taoyuan City 333, Taiwan; chengcchj@gmail.com (C.-H.C.); mp0616@cgmh.org.tw (H.-C.H.); \\ chiaopee@gmail.com (J.-C.L.); awuang726@gmail.com (Y.-C.W.); wutsunghan@gmail.com (T.-H.W.); \\ wutj5056@gmail.com (T.-J.W.); chouhs@cgmh.org.tw (H.-S.C.); chankunming@cgmh.org.tw (K.-M.C.); \\ weichen@cgmh.org.tw (W.-C.L.) \\ 2 College of Medicine, Chang-Gung University, Taoyuan City 333, Taiwan \\ * Correspondence: chenfanglee@gmail.com; Tel.: +886-3-3281200 (ext. 3366); Fax: +886-3-3285818
}

Citation: Lee, C.-F.; Cheng, C.-H.; Hung, H.-C.; Lee, J.-C.; Wang, Y.-C.; Wu, T.-H.; Wu, T.-J.; Chou, H.-S.; Chan, K.-M.; Lee, W.-C. Sedative and Immunosuppressive Effects of Dexmedetomidine in Transplantation. Pharmaceuticals 2021, 14, 825. https:// doi.org/10.3390/ph14080825

Academic Editor: Francois Dufrasne

Received: 28 July 2021

Accepted: 19 August 2021

Published: 22 August 2021

Publisher's Note: MDPI stays neutral with regard to jurisdictional claims in published maps and institutional affiliations.

Copyright: () 2021 by the authors. Licensee MDPI, Basel, Switzerland. This article is an open access article distributed under the terms and conditions of the Creative Commons Attribution (CC BY) license (https:// creativecommons.org/licenses/by/ $4.0 /)$.

\begin{abstract}
Dexmedetomidine, an $\alpha 2$-adrenergic receptor agonist, is used as an anti-anxiety medication. It exerts a cholinergic effect, thereby reducing the release of tumor necrosis factor alpha (TNF- $\alpha$ ). We hypothesized that the use of dexmedetomidine as a sedative agent in transplantation would also protect allografts. We examined our patients who underwent living donor liver transplantation. Subsequently, we generated a series of mouse models to investigate the effect of dexmedetomidine on sedation-based tolerance post transplantation. A total of 49 liver recipients were enrolled in this study, of which $23(47 \%)$ were administered dexmedetomidine through $24 \mathrm{~h}$ infusion on postoperative day 1. A trend toward the improvement of hepatocyte injury along with better liver function was observed in the dexmedetomidine-treated group during the first postoperative week. In animal models, dexmedetomidine inhibited the proliferation of $\mathrm{CD} 4^{+}$and $\mathrm{CD} 8^{+} \mathrm{T}$ cells and TNF- $\alpha$ production in a dose-dependent manner. We used dexmedetomidine to treat skin-transplanted mice and observed a significantly prolonged graft survival in mice that were administered a higher dose of dexmedetomidine. Our results revealed that dexmedetomidine exerts a dual effect of sedation and immunosuppression. This light-sedation approach will not only make patients calmer in the intensive care unit but also protect allografts from injury.
\end{abstract}

Keywords: delirium; tolerance; sedation; liver transplant; outcome

\section{Introduction}

Patients who undergo liver transplantation (LT) are at a high risk of delirium in the intensive care unit (ICU) [1,2]. The reported frequency of delirium in these recipients ranges from $12.7 \%$ to $47 \%$ [2]. Since delirium causes more complications and leads to longer stays in the ICU, it is important to identify patients who are at a higher risk and provide adequate sedation.

Dexmedetomidine, an $\alpha_{2}$-adrenergic receptor agonist, is used as an anti-anxiety medication and analgesic [3]. Since its sedative effect is mediated via the non- $\gamma$-aminobutyric acid (GABA) pathway, dexmedetomidine is thought to be less delirium-inducing and induce a more natural sleep cycle without the risk of respiratory depression [4]. The use of dexmedetomidine to prevent delirium in patients with LT or critical illness has been extensively recommended [1,5-7].

Some studies have reported that the electrical stimulation of the vagus nerve in animals activates choline acetyltransferase-positive $\mathrm{T}$ cells to secrete acetylcholine in the spleen and other tissues [8-10]. They also revealed that the stimulation of the vagus nerve inhibits TNF- $\alpha$ and attenuates disease severity in autoimmune disorders, such as rheumatoid arthritis and Crohn's disease [9,10]. Interestingly, recent studies have also demonstrated 
that dexmedetomidine exerts a cholinergic effect that helps reduce the release of the proinflammatory protein TNF- $\alpha[11,12]$. It has been shown to exhibit anti-inflammatory properties through promoting macrophage phagocytosis and bactericidal activity and reducing the levels of TNF- $\alpha$, IL-1, and IL-6 in patients with sepsis [11]. These immunological findings have attracted researchers' interest in assessing the effects of dexmedetomidine in major operations or transplantation. Indeed, the intraoperative infusion of dexmedetomidine has been shown to reduce ischemia-reperfusion injury during hepatectomy and liver transplantation [13,14], indicating its ability to reduce inflammation and immune modulation during operations. However, the impact of dexmedetomidine on LT in the early post-transplant period or long-term graft survival is unknown.

In this study, we hypothesized that the application of dexmedetomidine as a sedative agent post-transplantation may also help avoid allograft rejection. To analyze the antiinflammatory and immunosuppressive effects of the post-transplant infusion of dexmedetomidine, we reviewed our data from LT recipients and designed a series of animal experiments, along with the generation of a mouse model of skin transplant to investigate the efficacy of dexmedetomidine treatment on sedation-based tolerance and the possible underlying mechanisms.

\section{Results}

2.1. Dexmedetomidine Reduces Reperfusion Injury and Prevents Allograft Rejection in Human Liver Transplantation

Since 2016, our institute has prescribed dexmedetomidine to prevent pain and aggravated delirium to patients after LT. We did not use dexmedetomidine during anesthesia but administered it through continuous infusion $(0.2 \mathrm{mcg} / \mathrm{kg} / \mathrm{h})$ to the recipients upon arrival to the ICU after operation unless they were hemodynamically unstable. The maximum infusion time was $24 \mathrm{~h}$. The infusion of dexmedetomidine was terminated without restarting in the event of bradycardia, hypotension, or severe allergic reactions. Sedation levels were assessed using the Richmond Agitation Sedation Scale (RASS) [15]. The infusion of dexmedetomidine was titrated to achieve sedation, which was primarily light (RASS score of 0-2). Patients who underwent LT before 2016 without the use of dexmedetomidine were included in the control group. Finally, a total of 49 adult-to-adult living donor liver transplantations performed between 2013 and 2019 were retrospectively enrolled in this study, of which $23(47 \%)$ were sedated using dexmedetomidine and $26(53 \%)$ were assigned to the control group. The comparison of demographic and in-hospital characteristics between the two groups is summarized in Table 1. There was no difference in the recipients' age and sex, donors' age and sex, disease severity, ascites, or graft-to-recipient weight ratio between the two groups. In the dexmedetomidine and control groups, most of the patients received the right lobe from their donors ( $95.7 \%$ vs. $92.3 \%, p>0.999)$, and the major cause of liver cirrhosis was identified as hepatitis B $(56.5 \%$ vs. $38.5 \%, p=0.206)$. The proportions of hepatocellular carcinoma of two groups were $39.1 \%$ and $34.6 \%, p=0.744$. We assessed the reduction rates of aspartate aminotransferase (AST) and alanine aminotransferase (ALT) on postoperative days (POD) 2 and 4. Although not statistically significant, a trend towards the decrease in liver transaminase levels was observed in the dexmedetomidine group. The patients in the dexmedetomidine group exhibited more reduction rates of AST and ALT than those in the control group on POD 2 (AST: $32.75 \% \pm 4.23 \%$ vs. $29.74 \% \pm 6.44 \%, p=0.873$; ALT: $0.14 \% \pm 0.47 \%$ vs. $0.016 \% \pm 0.01 \%, p=0.317$.) and POD 4 (AST: $0.637 \% \pm 0.045 \%$ vs. $0.636 \% \pm 0.081 \%, p=0.873$; ALT: $0.39 \% \pm 0.08 \%$ vs. $0.25 \% \pm 0.01 \%, p=0.087$ ). The dexmedetomidine group also exhibited better liver function on POD 7: total bilirubin ( $4.93 \pm 1.45$ vs. $5.55 \pm 1.5 \mathrm{mg} / \mathrm{dL}, p=0.96)$, and international normalized ratio (INR) $(1.3 \pm 0.036$ vs. $1.2332 \pm 0.034, p=0.148)$. These results indicate that dexmedetomidine has the potential to reduce liver injury. 
Table 1. The comparisons of demographic and in-hospital characteristics between the two groups.

\begin{tabular}{lccc}
\hline Factors & $\begin{array}{c}\text { Dexmedetomidine } \\
\boldsymbol{n}=\mathbf{2 3}\end{array}$ & $\begin{array}{c}\text { Control } \\
\boldsymbol{n}=\mathbf{2 6}\end{array}$ & $\boldsymbol{p}$-Value \\
\hline General information & & & \\
Recipient age, median & $53.0(8.0)$ & $58.5(15.0)$ & 0.179 \\
Recipient gender (male) (\%) & $16(69.6)$ & $15(57.7)$ & 0.390 \\
Donor age, median & $31.0(20.0)$ & $28.0(10.0)$ & 0.527 \\
Donor gender (male) (\%) & $10(43.5)$ & $9(34.6)$ & 0.525 \\
Right lobe (\%) & $22(95.7)$ & $24(92.3)$ & $>0.999$ \\
MELD score, median & $14(8)$ & $16(13)$ & 0.166 \\
HBV infection (\%) & $13(56.5)$ & $10(38.5)$ & 0.206 \\
HCV infection (\%) & $3(13.0)$ & $10(38.5)$ & 0.044 \\
Alcohol use (\%) & $5(21.7)$ & $9(34.6)$ & 0.319 \\
HCC (\%) & $9(39.1)$ & $9(34.6)$ & 0.744 \\
Ascites (mL), median & $350.0(3200.0)$ & $950.0(2900.0)$ & 0.379 \\
GRWR (\%), median & $0.8(0.5)$ & $0.9(0.5)$ & 0.726 \\
Clinical outcomes & & & \\
AST POD2 reduction (\%), median & $32.75(4.23)$ & $29.74(6.44)$ & 0.873 \\
ALT POD2 reduction (\%), median & $0.14(0.47)$ & $0.016(0.01)$ & 0.317 \\
AST POD4 reduction (\%), median & $0.637(0.045)$ & $0.636(0.081)$ & 0.873 \\
ALT POD4 reduction (\%), median & $0.39(0.08)$ & $0.25(0.01)$ & 0.087 \\
Acute rejection (\%) & $13(56.5)$ & $17(68.0)$ & 0.412 \\
Total bilirubin (mg/dL) POD7, median & $4.93(1.45)$ & $5.55(1.5)$ & 0.96 \\
INR, median & $1.3(0.036)$ & $1.23(0.034)$ & 0.148 \\
ICU stay (day), median & $12.0(4.0)$ & $13.0(3.0)$ & 0.035 \\
Physical restraint time (min), median & $780.0(273.0)$ & $1005.0(1348.0)$ & 0.005 \\
\hline Abbration & & \\
\hline
\end{tabular}

Abbreviation: AST, aspartate transaminase; ALT, alanine aminotransferase; GRWR, graft recipient weight ratio $\mathrm{HBV}$, hepatitis B virus; $\mathrm{HCC}$, hepatocellular carcinoma; $\mathrm{HCV}$, hepatitis $\mathrm{C}$ virus; ICU, intensive care unit; INR, international normalized ratio; MELD, model for end-stage liver disease; POD, postoperative day.

Regarding the sedative effect, we used the confusion assessment method (CAM) [16] for the assessment of delirium; however, only a few delirium cases were identified in our cohort. Therefore, we recorded the total physical restraint time as an indicator of patients' levels of consciousness and compliance. Physical restraints were used to maintain the patients' device and therapy postoperatively. Once the patients were judged to be cooperative without delirium, the restraints were removed. The total physical restraint time for patients in the dexmedetomidine group was found to be significantly decreased (780 \pm 273 vs. $1005 \pm 1348 \mathrm{~min}, p=0.005$, Figure 1A), which means that dexmedetomidine made our patients calmer and minimized the reliance on restraint in critical care settings. The length of stay in the ICU was also significantly reduced in the dexmedetomidine group ( $12 \pm 4$ vs. $13 \pm 3$ days, $p=0.035$, Figure $1 B$ ), indicating more uneventful courses. We found no significant difference in the overall survival (OS) between the two groups. The 1-, $2-$, and 3 -year OS rates were $95.7 \%$ vs. $88.5 \%, 91.4 \%$ vs. $76.9 \%$, and $87 \%$ vs. $76.9 \%$ in the dexmedetomidine and control groups, respectively ( $p=0.538$, Figure 2$)$. 
A

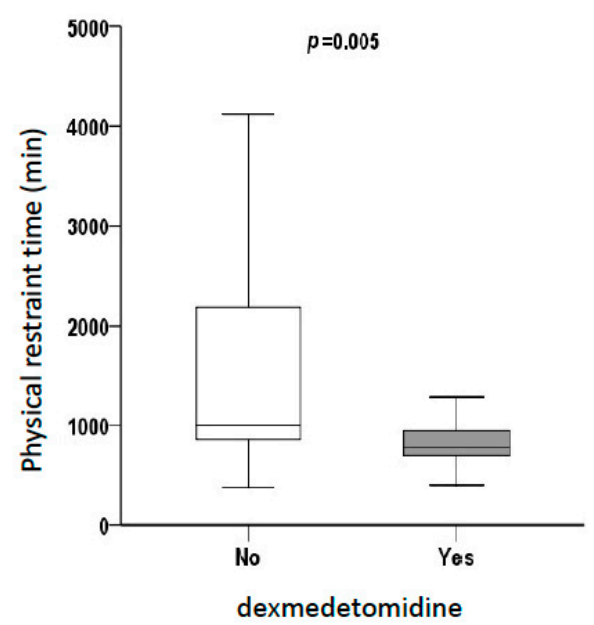

B

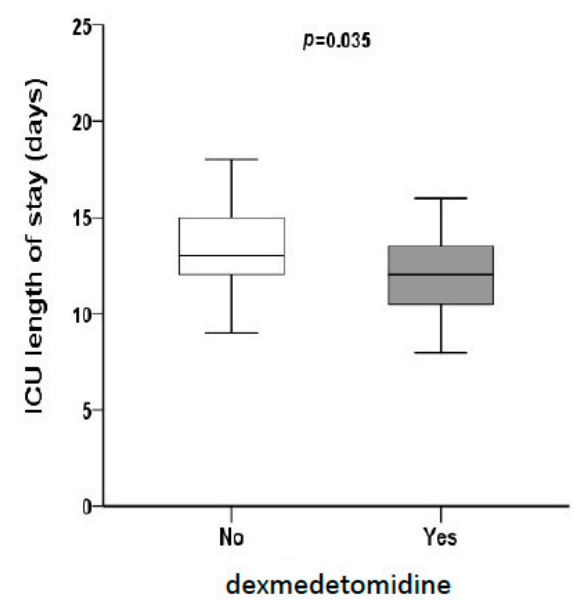

Figure 1. The dexmedetomidine group patients exhibited higher compliance and a shorter length of stay in the intensive care unit (ICU). (A) Total physical restraint time for patients in the dexmedetomidine group was significantly decreased (780 \pm 273 vs. $1005 \pm 1348 \mathrm{~min}, p=0.005)$. (B) The length of stay in the ICU was also significantly decreased in the dexmedetomidine group (12 \pm 4 vs. $13 \pm 3$ days, $p=0.035$ ).

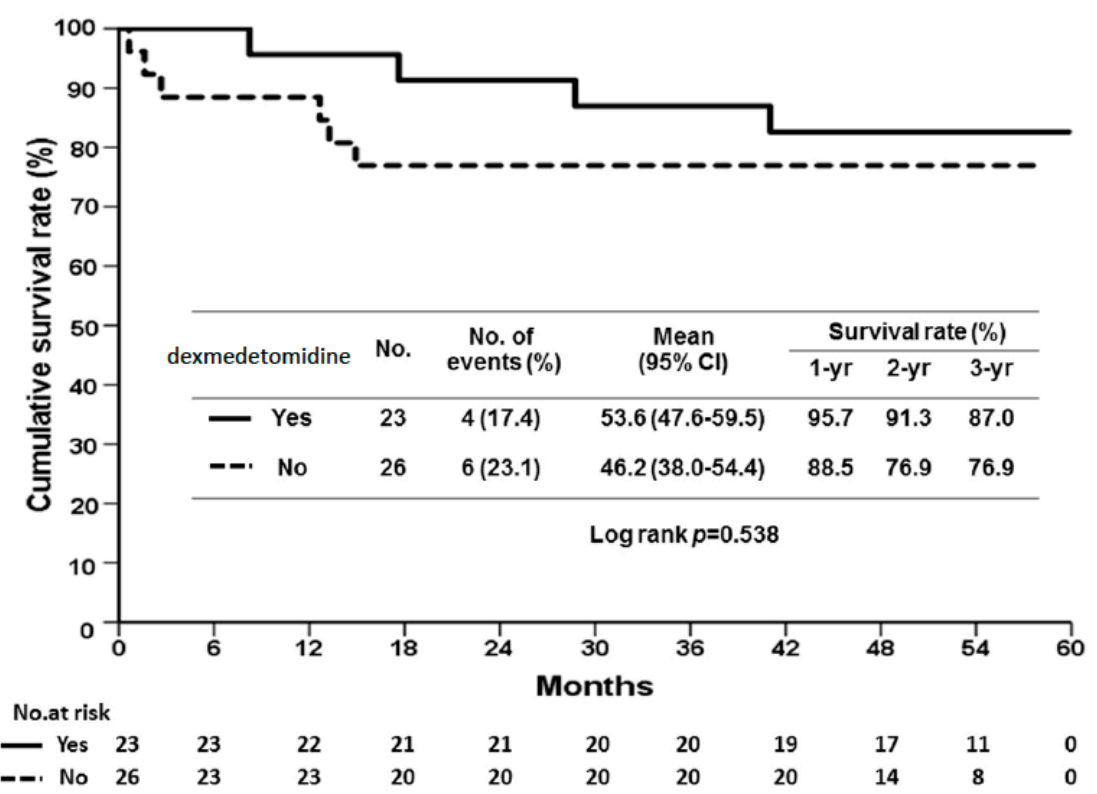

Figure 2. Comparisons of overall survival (OS) between dexmedetomidine and control groups. There was no significant difference in the overall survival (OS) between the two groups. The 1-, 2-, and 3-year OS rates were $95.7 \%$ vs. $88.5 \%, 91.4 \%$ vs. $76.9 \%$, and $87 \%$ vs. $76.9 \%$ ( $p=0.538$, log-rank analysis) in the dexmedetomidine and control groups, respectively.

Based on the above-mentioned results, we hypothesized that dexmedetomidine may exhibit the potential to not only prevent delirium but also to improve hepatocyte injury after LT. However, the $24 \mathrm{~h}$ infusion of dexmedetomidine may not be sufficient to achieve significant differences in avoiding reperfusion injury and allograft rejection. Therefore, we then used a mouse full-thickness skin transplant model to better characterize the effects of dexmedetomidine on sedation-based tolerance and dissect the possible underlying mechanisms.

\subsection{Dexmedetomidine Abrogates Activation-Induced T-Cell Proliferation and Cytokine Production}

First, we sought to determine the effect of dexmedetomidine on $\mathrm{CD}^{+}$and $\mathrm{CD} 8^{+} \mathrm{T}$-cell proliferation. To this end, a titration dose (from 100 to $250 \mu \mathrm{g} / \mathrm{kg}$ ) of dexmedetomidine 
was injected into the peritoneal cavity of C57BL/6 mice twice a day. Three days later, naïve T cells were harvested from the spleen and stimulated with anti-CD3 $(1 \mu \mathrm{g} / \mathrm{mL})$ in the culture medium for $48 \mathrm{~h}$. The proliferation of $\mathrm{CD} 4^{+} / \mathrm{CD}^{+}$cells was assessed by the dilution of cell proliferation dye carboxyfluorescein succinimidyl ester (CFSE). The results of these titration studies demonstrated that dexmedetomidine markedly inhibited $\mathrm{CD} 4^{+}$ and $\mathrm{CD}^{+} \mathrm{T}$-cell proliferation in a dose-dependent manner (Figure 3)
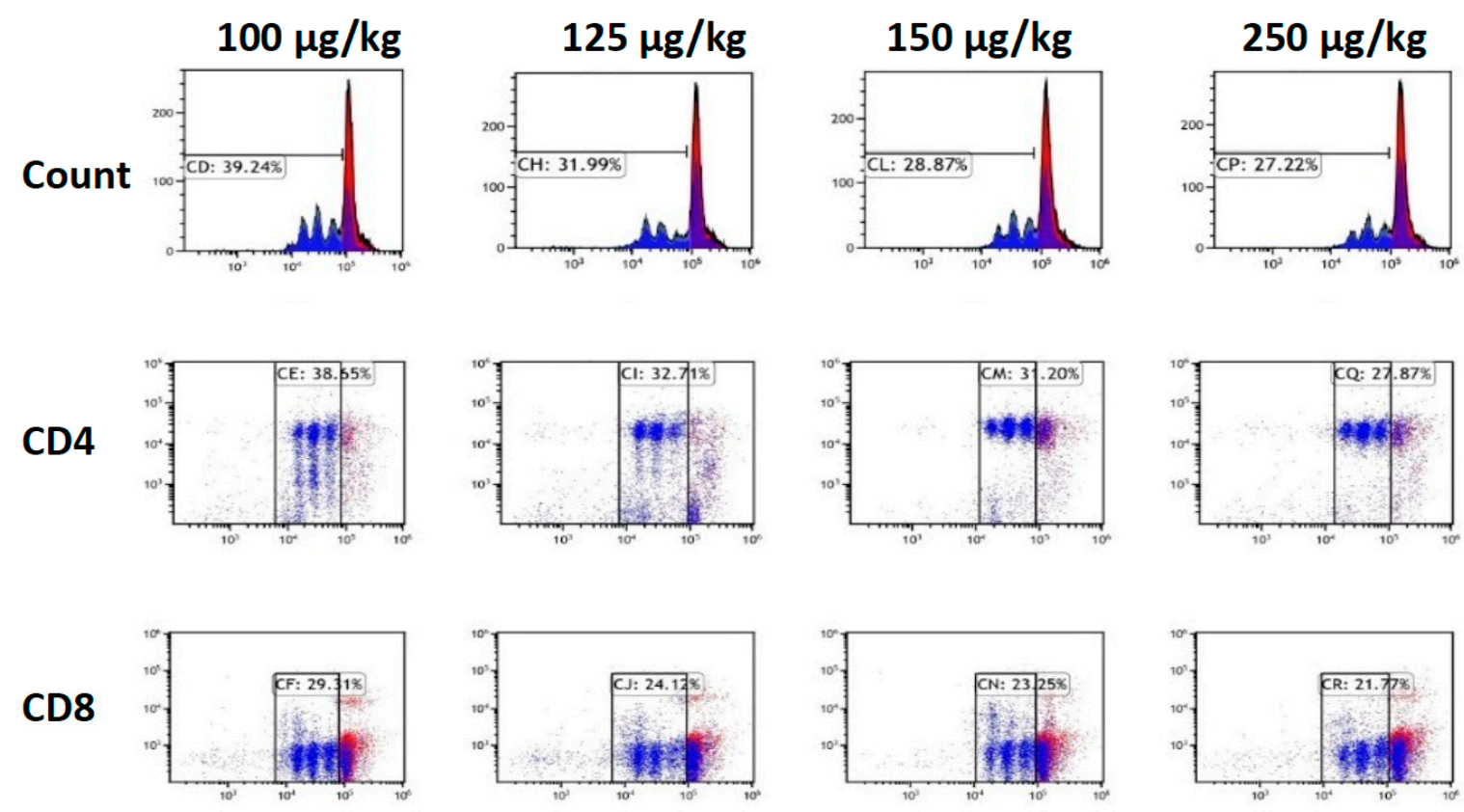

Figure 3. Dexmedetomidine abrogates activation-induced T-cell proliferation. Naïve splenocytes from wide-type C57BL/6 mice labeled with cell proliferation dye carboxyfluorescein succinimidyl ester (CFSE) were stimulated with anti-CD3 $(1 \mu \mathrm{g} / \mathrm{mL})$ after the treatment with dexmedetomidine intraperitoneally with titration concentrations twice a day for $48 \mathrm{~h}$. The proliferation of $\mathrm{CD}^{+} / \mathrm{CD}^{+}$was assessed upon the dilution of CFSE. The results of these titration studies demonstrated that dexmedetomidine inhibited $\mathrm{CD}^{+}$and $\mathrm{CD} 8^{+} \mathrm{T}$-cell proliferation in a dose-dependent manner. Data are representative of three independent experiments.

Next, an in vitro model was used to further investigate the suppressive effect of dexmedetomidine on T-cell activation; splenocytes from wild-type C57BL/ 6 mice were stimulated with anti-CD3 $(1 \mu \mathrm{g} / \mathrm{mL})$ and cultured in the presence of titration concentrations of dexmedetomidine for $48 \mathrm{~h}$. The expression of the activation markers, CD44 and CD25, in CFSE-labeled T cells at $48 \mathrm{~h}$ was evaluated using flow cytometry analysis. We found that dexmedetomidine suppressed the expression of both CD44 and CD25 and inhibited T-cell proliferation in a dosedependent manner (Figure 4A). To test the ability of dexmedetomidine to mitigate cytokine production, the TNF- $\alpha$ concentration in the supernatant of cell cultures was evaluated using enzyme-linked immunosorbent assay (ELISA). As a result, the marked inhibition of TNF- $\alpha$ production was observed, as shown in Figure 4B. Notably, the decrease in $\mathrm{CD}^{+}$and $\mathrm{CD} 8^{+}$ cell function was not due to a decrease in cell viability (data not shown). Together, these data suggest that dexmedetomidine potently inhibits T-cell activation and proliferation. 
A
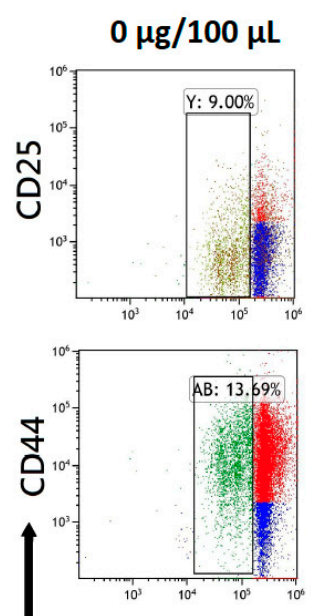

$10 \mu \mathrm{g} / 100 \mu \mathrm{L}$
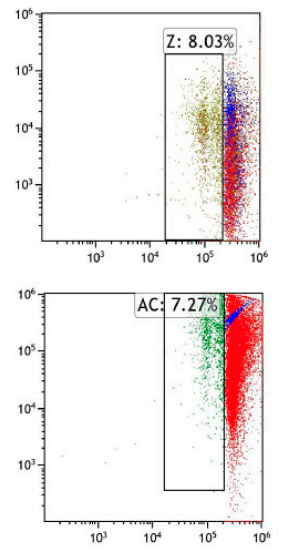

CFSE
B
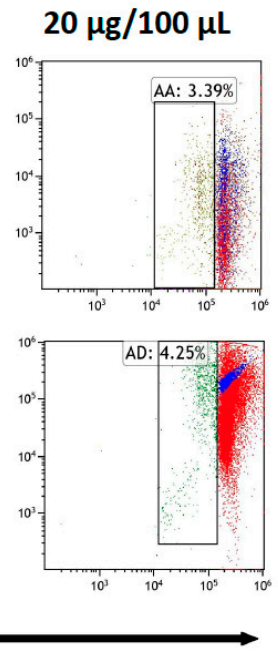

TNF- $\alpha(\mathrm{pg} / \mathrm{mL})$

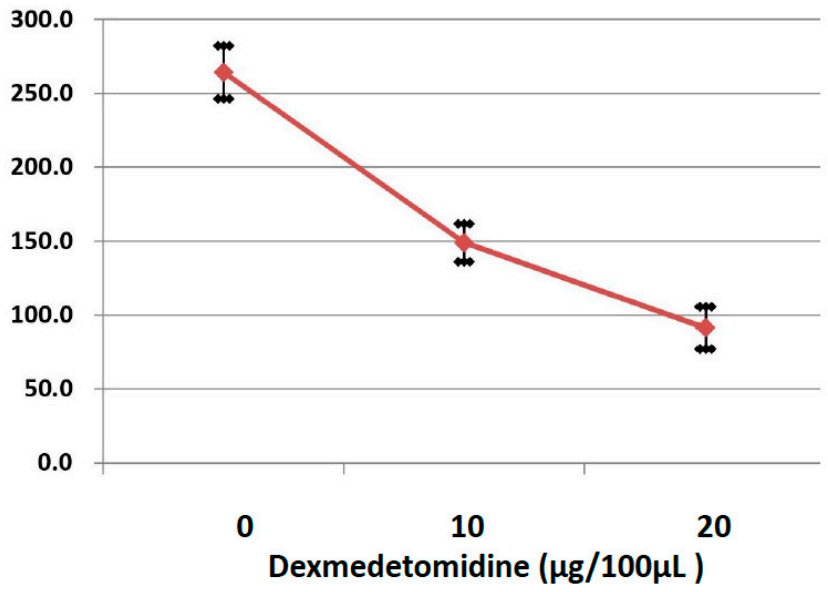

Figure 4. Dexmedetomidine inhibits T-cell activation and cytokine production. Splenocytes from wide-type C57BL/6 mice were stimulated with anti-CD3 $(1 \mu \mathrm{g} / \mathrm{mL})$ and cultured in the presence of dexmedetomidine with titration concentrations. (A) The expression of the activation markers, CD44 and CD25, in viable T cells at $48 \mathrm{~h}$. Data are representative of two independent experiments. (B) TNF- $\alpha$ secretion in the supernatant was examined using enzyme-linked immunosorbent assay (ELISA). These data suggest that dexmedetomidine inactivates T cells. Data are shown as mean \pm SEM of three independent samples.

\subsection{Dexmedetomidine Promotes Allograft Acceptance in Mouse Models of Skin Transplantation}

After demonstrating the ability of dexmedetomidine to inhibit T-cell proliferation and activation, we next investigated whether it could prolong the allograft survival using a fully MHC-mismatched skin transplantation model involving C57BL/6 mice that received allogenic skin grafts from BALB/c mice. We employed dexmedetomidine in titration doses (from 0 to $250 \mu \mathrm{g} / \mathrm{kg}$ ) intraperitoneally twice a day to treat the skin-transplanted mice and examined the median survival time (MST) of skin grafts. We found significantly prolonged graft survival in mice that received a higher dose of dexmedetomidine compared to those that received a lower dose or no treatment (all $p<0.05$, Figure $5 \mathrm{~A}, \mathrm{~B}$ ). The grafts were monitored daily, and the macroscopic appearance of skin grafts in the higher dose group exhibited less tissue destruction and much healthier alignment. The representative photographs of the skin grafts at POD 11 are presented in Figure 5C. These data further support the idea that sedation-based immunosuppression can be effectively applied to prolong allograft survival. 
A

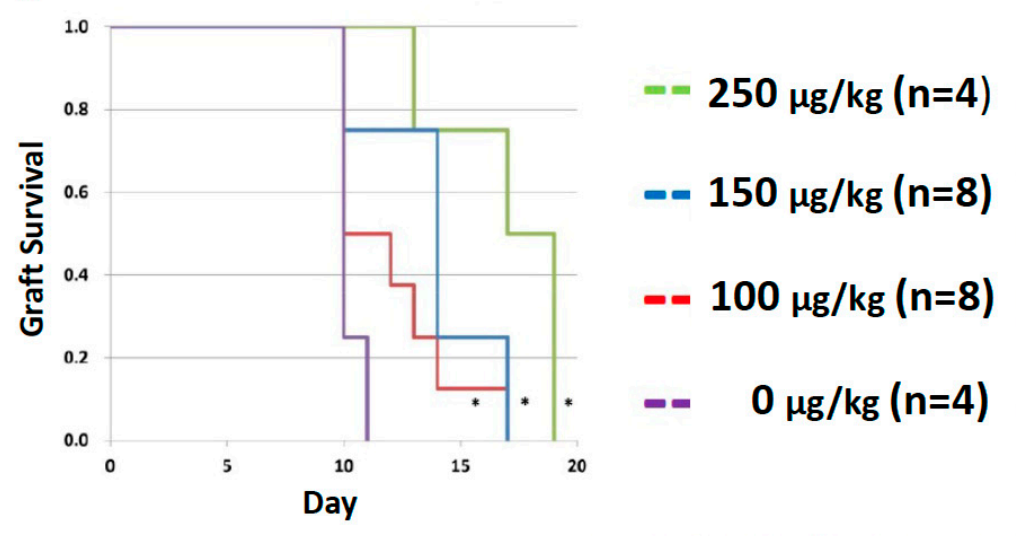

B

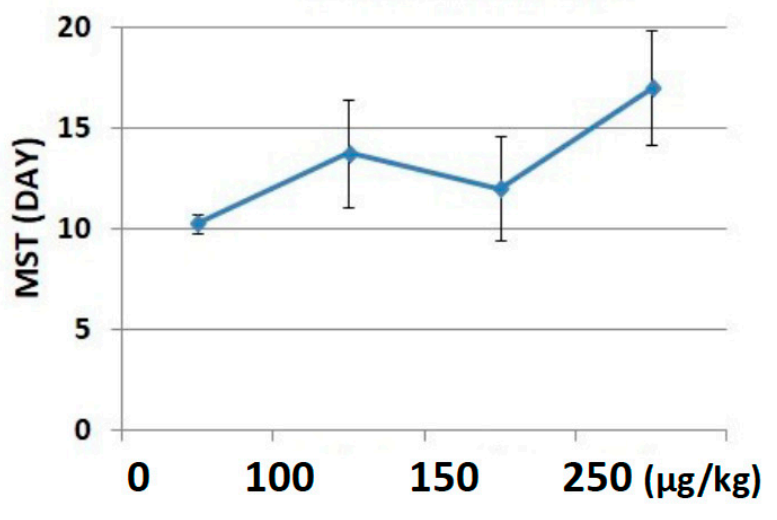

C

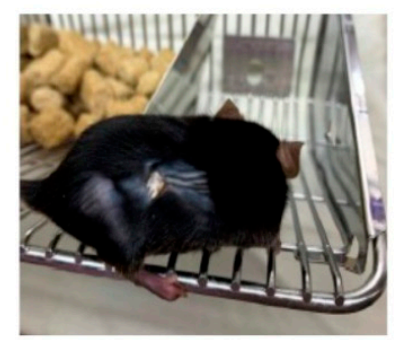

$0 \mu \mathrm{g} / \mathrm{kg}$

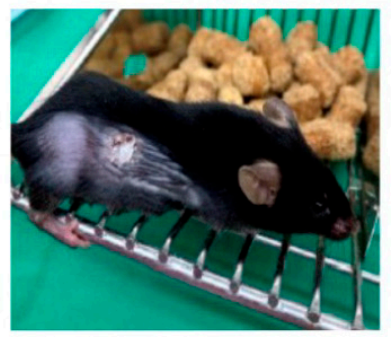

$100 \mu \mathrm{g} / \mathrm{kg}$

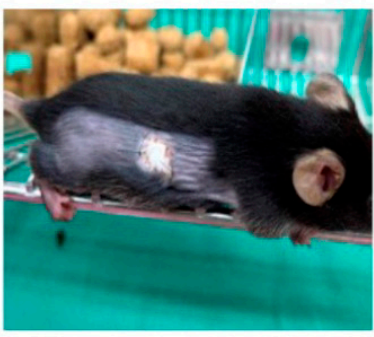

$150 \mu \mathrm{g} / \mathrm{kg}$

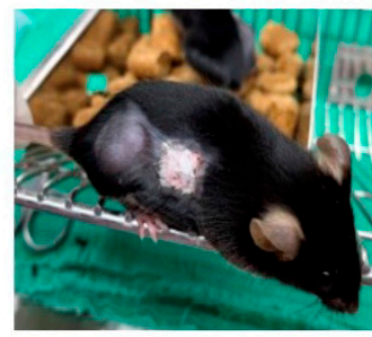

$250 \mu \mathrm{g} / \mathrm{kg}$

Figure 5. Dexmedetomidine promotes allograft acceptance in mouse models of skin transplantation. (A,B) Dexmedetomidine was used to treat the skin-transplanted C57BL/ 6 mice intraperitoneally twice a day. There was a significant increase in the median survival time (MST) compared to those that received no treatment ( ${ }^{*}$ all $p<0.05$, log-rank analysis). (C) Representative photographs of the skin grafts at postoperative day 11. Data are representative of two independent experiments.

\section{Discussion}

In this study, we designed a light-sedation experiment to prevent allograft rejection using dexmedetomidine that inhibits T-cell proliferation and activation. Specifically, the significance and major findings of our study involved the targeting of cholinergic anti-inflammatory pathways using dexmedetomidine as an effective agent to mitigate $\mathrm{CD}^{+} / \mathrm{CD}^{+} \mathrm{T}$-cell proliferation and activation, thereby avoiding acute allograft rejection. This study is based on the findings that dexmedetomidine, an $\alpha_{2}$-agonist, reduces the release of TNF- $\alpha$ and prevents both delirium and hepatocyte injury in human liver transplantation $[1,13]$. Although a postoperative $24 \mathrm{~h}$ continuous infusion of dexmedetomidine did not result in significant differences in the incidence of rejection and overall survival, the dexmedetomidine group patients exhibited higher compliance and reduced length of stay in the ICU. Patients in the dexmedetomidine group also exhibited higher reduction rates in the liver transaminase levels than those in the control group, thereby indicating the ability of dexmedetomidine to exert protective effects against inflammation and liver allograft rejection. To gain a better understanding of this therapeutic potential, a fully MHC-mismatched mouse skin transplantation model was used to assess the efficacy of dexmedetomidine on sedation-based tolerance. Through our animal model, we demonstrated that the continuous administration of dexmedetomidine may inhibit T-cell activation and result in prolonged skin allograft survival.

The link between sedation and immunity is now being recognized. Recent advances in the interaction between immunology and neuroscience have revealed the reflex neural circuit mechanisms that regulate both innate and adaptive immunity $[17,18]$. One such wellcharacterized reflex circuit, termed the "inflammatory reflex", is defined by the signals that transmit through the vagus nerve to inhibit the monocyte and macrophage production of TNF- $\alpha$ and other cytokines [17]. The vagus nerve is a mixed nerve, which exhibits antiinflammatory properties through the activation of the hypothalamic-pituitary-adrenal axis 
by inducing the cholinergic anti-inflammatory pathway. Therefore, vagal nerve stimulation can be used to alleviate autoimmune disorders $[9,10]$. The underlying mechanism of dexmedetomidine-mediated anti-inflammation or the tolerance effect is thought to operate through a similar link between the immune and nervous systems. Xiang et al. demonstrated that the central $\alpha-2$ agonist, dexmedetomidine, suppresses systemic inflammation through vagal- and $\alpha-7$ nicotinic acetylcholine receptor-dependent mechanisms [19]. They found that dexmedetomidine increases the activity of the cervical vagus nerve and improves the survival in experimental endotoxemia through inhibiting the inflammatory cytokines [19]. Along these lines, we believe that the targeting of sedation-induced immunosuppression may provide a therapeutic opportunity to simultaneously inhibit delirium and promote tolerance.

Immunosuppression may also increase the susceptibility to microbial colonization. In our animal study, we followed weight and fur ruffling as the markers of overall health and did not observe any morbidity associated with the treatment regimen. Although the pharmacokinetics of dexmedetomidine are largely influenced by the liver rather than renal function [20], its administration did not worsen the hepatic or renal functions in our recipients, when compared to the subjects in the control group. However, if we do observe inappropriate effects, this may indicate the need to adjust the dose and schedule of the treatment regimen. Conversely, extending or increasing the administration of dexmedetomidine may significantly reduce reperfusion injury and avoid rejection in human LT.

The long-term use of immunosuppressants leads to a broad range of comorbidities. Such regimens markedly increase the susceptibility to infections and block the induction of tolerance. As such, new regimens or adjuvants devoid of these side effects that promote immunologic tolerance are always considered superior [21,22]. However, in our model, it was not sufficient to induce tolerance. To improve this effect, in our future work, we aim to strategically include tolerance-inducing drugs, for example, mTOR inhibitor rapamycin or costimulatory blockade, in combination with dexmedetomidine to promote long-term allograft acceptance [23].

This study has some limitations. First, the retrospective design in a single center restricted the sample size, and protocol bias may arise. Second, there might be a practical gap between basic research and clinical application. Unlike solid organ transplantation, mechanisms regulating ischemia-reperfusion injury in vascularized composite allotransplantation, such as the skin, are not well elucidated. In addition, their unique immunological structures indicate that the comparability of the two models presented may not be pertinent. Therefore, the influence of dexmedetomidine on effector T-cell function and differentiation needs to be further investigated and addressed in the future. Larger and prospective human studies are required to confirm these results.

\section{Materials and Methods}

\subsection{Human Liver Transplantation}

\subsubsection{Data Collection of Human Liver Transplantation}

The clinical data, including age, sex, model for end-stage liver disease scores, causes of LT, postoperative liver function, reduction rates in hepatic transaminase (including AST and ALT) on POD 2 and 4, physical restraint time, length of stay in the ICU, and overall survival were compared between the two groups. The reduction rate of the transaminase level was calculated as follows: \{(transaminase level on POD1-transaminase level on POD2)/transaminase level on POD1 $\} \times 100 \%$ and $\{$ (transaminase level on POD1transaminase level on POD4)/transaminase level on POD1\} $\times 100 \%$. Higher reduction rates represented fewer liver allograft injuries. The protocol of this retrospective study was approved by the Ethics Committee and IRB of Chang Gung Memorial Hospital (approval no. 201900976B0) and conformed to the ethical guidelines of the 1975 Declaration of Helsinki. 


\subsubsection{Immunosuppression Protocol and Surgical Techniques in Liver Transplantation}

For both groups, regular immunosuppression using tacrolimus, steroids, and mycophenolate mofetil was prescribed. Methylprednisolone administration was initiated immediately after LT. Tacrolimus was introduced and adjusted according to the morning trough level on POD 1. Mycophenolate mofetil was administered from POD 14-21 (daily doses of $2 \mathrm{~g}$ ). The details of the immunosuppression protocol and standard surgical techniques used in this study have been described previously [24-26]. We do not routinely remove the spleen and preserve the middle hepatic vein for donors.

\subsection{Animal Model}

Male C57BL/6 (Thy1.2 $2^{+}$H-2 $\left.{ }^{\mathrm{b}}\right)$ and BALB/c $\left(\mathrm{H}-2^{\mathrm{d}}\right)$ mice of 6 to 8 weeks old were purchased from the Animal Laboratory of National Institute, Taipei, Taiwan. The mice were maintained in the pathogen-free facility and were kept in accordance with the guidelines of the Animal Care Committee. Animals had free access to natural ingredient diets and water in common cages. In this non-blinded study, mice were randomly assigned to different treatment conditions. The use of these mice for experimental purposes was approved by the Chang Gung Memorial Hospital Animal Care and Use Committee.

\subsection{Cell Culture, T-Cell Activation, and Cytokine Production}

To investigate the role of dexmedetomidine in T-cell activation, naïve $\mathrm{CD} 4^{+} \mathrm{T}$ cells were stimulated with anti-CD3/CD28 in the medium with different doses of dexmedetomidine for 48 h. Cells were cultured in 45\% RPMI 1640 and 45\% Eagle Hank's amino acid medium supplemented with $10 \%$ fetal bovine serum and antibiotics. For proliferation studies, $\mathrm{T}$ cells were labeled with CFSE. Dexmedetomidine was purchased from Pfizer (Brooklyn, NY, USA).

\subsection{Flow Cytometry and Intracellular Cytokine Staining}

Flow cytometry data were acquired using the BD FACS Calibur (New Jersey, USA). Cells were surface-stained. Gates were determined appropriately using unstimulated control cells. Voltage was determined using unstained controls. TNF- $\alpha$ concentration in the supernatant of cell cultures was analyzed using ELISA, according to the manufacturer's instructions. Cell viability was determined via propidium iodide and annexin V staining.

\subsection{Murine Skin Transplantation Model}

Full-thickness skin grafts were harvested from BALB/c mice and transplanted onto the thoracic flank of recipient C57BL/ 6 mice with simple separate stitches. The size of the transplanted grafts was $1 \times 1 \mathrm{~cm}^{2}$. The grafts were monitored daily and were considered as rejected when more than $90 \%$ of the graft tissue turned necrotic [27]. The full-thickness skin transplantation was performed under the following conditions: 1 . No treatment group: positive control for rapid rejection. 2. Dexmedetomidine group: to determine if this agent exerts any effects in terms of promoting graft survival. We compared the median survival time of skin grafts in each group.

\subsection{Statistical Considerations and Analysis}

Significant differences between means were determined using an unpaired Student's $t$-test, two-way analysis of variance, and log-rank analysis. The independent Student's t-test was used to determine the relationships between continuous variables (expressed as mean \pm standard deviation), while Pearson's Chi-squared test was used for categorical variables (numbers and percentages). The Mann-Whitney $U$ test was used to analyze the non-parametric data. Survival comparisons were performed using the Kaplan-Meier method with the log-rank test. All $p$-values were two-sided, and a level $p \leq 0.05$ was considered statistically significant. All analyses were performed using SPSS software (version 24.0; SPSS Inc., Chicago, IL, USA). 


\section{Conclusions}

Conclusively, the main finding of this study is that the targeting of sedation-based immunosuppression is an effective approach to transplantation. This light-sedation approach will not only make the recipients more cooperative and calmer in the ICU, but also protect allografts from injury and rejection. The immunosuppression effects of such sedatives will play a critical role in the field of transplantation, and their use may be designed toward therapeutic manipulation of the immune response. These results highlight new strategies for potentially inducing long-term transplant tolerance and survival.

Author Contributions: Conceptualization, C.-F.L. and C.-H.C.; methodology, C.-F.L. and C.-H.C., and H.-C.H.; software, H.-C.H. and J.-C.L.; resources, T.-H.W., Y.-C.W.; data curation, T.-H.W., Y.C.W., and T.-J.W.; writing—original draft preparation, C.-F.L.; writing—review and editing, C.-F.L.; visualization, H.-S.C.; supervision, H.-S.C., K.-M.C. and W.-C.L.; funding acquisition, C.-F.L. All authors have read and agreed to the published version of the manuscript.

Funding: This study was supported by a research grant of Chang-Gung Memorial Hospital CMRPG3J1401.

Institutional Review Board Statement: The study was conducted according to the guidelines of the Declaration of Helsinki and approved by the Institutional Review Board of Chang-Gung Memorial Hospital (IRB No. 201900976B0).

Informed Consent Statement: Patients were not required to give informed consent to the study because the analysis used anonymous clinical data that were obtained after each patient agreed to treatment by written consent.

Data Availability Statement: All data are contained within the article.

Acknowledgments: We thank Hsin-Yu Hsu and members of Wei-Chen Lee's lab for help in this study.

Conflicts of Interest: The authors declare no conflict of interest.

$\begin{array}{ll}\text { Abbreviations } \\ \text { AST } & \text { Aspartate aminotransferase } \\ \text { ALT } & \text { Alanine aminotransferase } \\ \text { CFSE } & \text { carboxyfluorescein succinimidyl ester } \\ \text { ELISA } & \text { enzyme-linked immunosorbent assay } \\ \text { GRWR } & \text { Graft-recipient weight ratio } \\ \text { HBV } & \text { Hepatitis B virus } \\ \text { HCC } & \text { Hepatocellular carcinoma } \\ \text { HCV } & \text { Hepatitis C virus } \\ \text { ICU } & \text { Intensive care unit } \\ \text { INR } & \text { International normalized ratio } \\ \text { LT } & \text { Liver transplantation } \\ \text { MELD } & \text { Model of end-stage liver disease } \\ \text { MST } & \text { Median survival time } \\ \text { OS } & \text { Overall survival } \\ \text { POD } & \text { Postoperative day } \\ \text { RASS } & \text { Richmond Agitation Sedation Scale }\end{array}$

\section{References}

1. Choi, J.Y.; Kim, J.M.; Kwon, C.H.; Joh, J.W.; Lee, S.; Park, J.B.; Ko, J.S.; Gwak, M.S.; Kim, G.S.; Kim, S.J.; et al. Use of Dexmedetomidine in Liver Transplant Recipients With Postoperative Agitated Delirium. Transplant. Proc. 2016, 48, 1063-1066. [CrossRef]

2. Lee, H.; Yang, S.M.; Chung, J.; Oh, H.W.; Yi, N.J.; Suh, K.S.; Oh, S.Y.; Ryu, H.G. Erratum to Effect of Perioperative Low-Dose Dexmedetomidine on Postoperative Delirium After Living-Donor Liver Transplantation: A Randomized Controlled Trial, by Hannah Lee, Seong Mi Yang, Jaeyeon Chung, Hye-Won Oh, Nam Joon Yi, Kyung-Suk Suh, Seung-Young Oh, and Ho Geol Ryu Transplant Proc. 2020; 52(1):239-245. Transplant. Proc. 2021, 53, 1771. 
3. Pandharipande, P.; Ely, E.W.; Maze, M. Alpha-2 agonists: Can they modify the outcomes in the Postanesthesia Care Unit? Curr. Drug Targets 2005, 6, 749-754. [CrossRef] [PubMed]

4. Nelson, L.E.; Lu, J.; Guo, T.; Saper, C.B.; Franks, N.P.; Maze, M. The alpha2-adrenoceptor agonist dexmedetomidine converges on an endogenous sleep-promoting pathway to exert its sedative effects. Anesthesiology 2003, 98, 428-436. [CrossRef]

5. Djaiani, G.; Silverton, N.; Fedorko, L.; Carroll, J.; Styra, R.; Rao, V.; Katznelson, R. Dexmedetomidine versus Propofol Sedation Reduces Delirium after Cardiac Surgery: A Randomized Controlled Trial. Anesthesiology 2016, 124, 362-368. [CrossRef] [PubMed]

6. Pasin, L.; Landoni, G.; Nardelli, P.; Belletti, A.; Di Prima, A.L.; Taddeo, D.; Isella, F.; Zangrillo, A. Dexmedetomidine reduces the risk of delirium, agitation and confusion in critically Ill patients: A meta-analysis of randomized controlled trials. J. Cardiothorac. Vasc. Anesth. 2014, 28, 1459-1466. [CrossRef]

7. Alexopoulou, C.; Kondili, E.; Diamantaki, E.; Psarologakis, C.; Kokkini, S.; Bolaki, M.; Georgopoulos, D. Effects of dexmedetomidine on sleep quality in critically ill patients: A pilot study. Anesthesiology 2014, 121, 801-807. [CrossRef]

8. Rosas-Ballina, M.; Olofsson, P.S.; Ochani, M.; Valdes-Ferrer, S.I.; Levine, Y.A.; Reardon, C.; Tusche, M.W.; Pavlov, V.A.; Andersson, U.; Chavan, S.; et al. Acetylcholine-synthesizing $\mathrm{T}$ cells relay neural signals in a vagus nerve circuit. Science 2011, 334, 98-101. [CrossRef]

9. Bonaz, B.; Sinniger, V.; Hoffmann, D.; Clarencon, D.; Mathieu, N.; Dantzer, C.; Vercueil, L.; Picq, C.; Trocmé, C.; Faure, P.; et al. Chronic vagus nerve stimulation in Crohn's disease: A 6-month follow-up pilot study. Neurogastroenterol. Motil. 2016, 28 , 948-953. [CrossRef]

10. Koopman, F.A.; Chavan, S.S.; Miljko, S.; Grazio, S.; Sokolovic, S.; Schuurman, P.R.; Mehta, A.D.; Levine, Y.A.; Faltys, M.; Zitnik, R.; et al. Vagus nerve stimulation inhibits cytokine production and attenuates disease severity in rheumatoid arthritis. Proc. Natl. Acad. Sci. USA 2016, 113, 8284-8289. [CrossRef]

11. MacLaren, R. Immunosedation: A consideration for sepsis. Crit. Care 2009, 13, 191. [CrossRef]

12. Jiang, W.W.; Wang, Q.H.; Liao, Y.J.; Peng, P.; Xu, M.; Yin, L.X. Effects of dexmedetomidine on TNF-alpha and interleukin-2 in serum of rats with severe craniocerebral injury. BMC Anesthesiol. 2017, 17, 130. [CrossRef]

13. Fayed, N.A.; Sayed, E.I.; Saleh, S.M.; Ehsan, N.A.; Elfert, A.Y. Effect of dexmedetomidine on hepatic ischemia-reperfusion injury in the setting of adult living donor liver transplantation. Clin. Transplant. 2016, 30, 470-482. [CrossRef]

14. Zhang, Y.; Liu, M.; Yang, Y.; Cao, J.; Mi, W. Dexmedetomidine exerts a protective effect on ischemia-reperfusion injury after hepatectomy: A prospective, randomized, controlled study. J. Clin. Anesth. 2020, 61, 109631. [CrossRef]

15. Sessler, C.N.; Gosnell, M.S.; Grap, M.J.; Brophy, G.M.; O’Neal, P.V.; Keane, K.A.; Tesoro, E.P.; Elswick, R.K. The Richmond Agitation-Sedation Scale: Validity and reliability in adult intensive care unit patients. Am. J. Respir. Crit. Care Med. 2002, 166, 1338-1344. [CrossRef]

16. Ely, E.W.; Inouye, S.K.; Bernard, G.R.; Gordon, S.; Francis, J.; May, L.; Truman, B.; Speroff, T.; Gautam, S.; Margolin, R.; et al. Delirium in mechanically ventilated patients: Validity and reliability of the confusion assessment method for the intensive care unit (CAM-ICU). JAMA 2001, 286, 2703-2710. [CrossRef] [PubMed]

17. Wu, T.J.; Dahiya, D.; Lee, C.S.; Lee, C.F.; Chou, H.S.; Chan, K.M.; Lee, W.C. Impact of portal venous hemodynamics on indices of liver function and graft regeneration after right lobe living donor liver transplantation. Liver Transplant. 2011, 17, 1035-1045. [CrossRef] [PubMed]

18. Chan, K.M.; Cheng, C.H.; Wu, T.H.; Wu, T.J.; Chou, H.S.; Lee, C.S.; Lee, W.C. Clinical strategy for the reconstruction of middle hepatic vein tributaries in right liver living donor liver transplantation. World J. Surg. 2014, 38, 2927-2933. [CrossRef]

19. Lee, C.F.; Cheng, C.H.; Wang, Y.C.; Soong, R.S.; Wu, T.H.; Chou, H.S.; Wu, T.J.; Chan, K.M.; Lee, C.S.; Lee, W.C. Adult Living Donor Liver Transplantation Across ABO-Incompatibility. Medicine 2015, 94, e1796. [CrossRef]

20. Cheng, C.H.; Lee, C.F.; Fryer, M.; Furtmuller, G.J.; Oh, B.; Powell, J.D.; Brandacher, G. Murine Full-thickness Skin Transplantation. J. Vis. Exp. 2017, 55105. [CrossRef]

21. Andersson, U.; Tracey, K.J. Neural reflexes in inflammation and immunity. J. Exp. Med. 2012, 209, 1057-1068. [CrossRef] [PubMed]

22. Andersson, U.; Tracey, K.J. Reflex principles of immunological homeostasis. Annu. Rev. Immunol. 2012, 30, 313-335. [CrossRef]

23. Xiang, H.; Hu, B.; Li, Z.; Li, J. Dexmedetomidine controls systemic cytokine levels through the cholinergic anti-inflammatory pathway. Inflammation 2014, 37, 1763-1770. [CrossRef] [PubMed]

24. Wang, L.; Wang, S.; Qi, J.; Yu, R.; Zhuang, J.; Zhuang, B.; Lou, Y.; Ruan, J.; Ye, H.; Lin, F. Impact of CYP2A6 gene polymorphism on the pharmacokinetics of dexmedetomidine for premedication. Expert Rev. Clin. Pharmacol. 2018, 11, 917-922. [CrossRef]

25. Lee, C.F.; Lo, Y.C.; Cheng, C.H.; Furtmuller, G.J.; Oh, B.; Andrade-Oliveira, V.; Thomas, A.G.; Bowman, C.E.; Slusher, B.S.; Wolfgang, M.J.; et al. Preventing Allograft Rejection by Targeting Immune Metabolism. Cell Rep. 2015, 13, 760-770. [CrossRef] [PubMed]

26. Lo, Y.C.; Lee, C.F.; Powell, J.D. Insight into the role of mTOR and metabolism in T cells reveals new potential approaches to preventing graft rejection. Curr. Opin. Organ Transplant. 2014, 19, 363-371. [CrossRef]

27. Cheng, C.H.; Lee, C.F.; Oh, B.C.; Furtmüller, G.J.; Patel, C.H.; Brandacher, G.; Powell, J.D. Targeting Metabolism as a Platform for Inducing Allograft Tolerance in the Absence of Long-Term Immunosuppression. Front. Immunol. 2020, 11, 572. [CrossRef] [PubMed] 